O BE PRESENTED AT THE 1995 PARTICLE ACCELERATOR CONFERENCE AND INTERNATIONAL CONFERENCE ON HIGH ENERGY ACCELERATORS DALLAS, TEXAS MAY 1-5, 1995

\title{
QUENCH ANTENNAS FOR RHIC QUADRUPOLE MAGNETS
}

\author{
Conf- 95
}

KEK, National Laboratory for High Energy Physics, Tsukuba, Ibaraki, 305, Japan

G. Ganetis, J. Muratore, and P. Wanderer,

BNL, Brookhaven National Laboratory, Upton, Long Island, NY 11973 USA

Quench antennas for RHIC quadrupole magnets are being developed jointly by KEK and BNL. A quench antenna is a device to localize a quench origin using arrays of pick-up coils lined up along the magnet bore. Each array contains four pick-up coils: sensitive to normal sextupole, skew sextupole, normal octupole, and skew octupole field. This array configuration allows an azimuthal localization of a quench front while a series of arrays gives an axial localization and a quench propagation velocity. Several antennas have been developed for RHIC magnets and they are now routinely used for quench tests of production magnets. The paper discusses the description of the method and introduces a measured example using an antenna designed for quadrupole magnets.

\section{INTRODUCTION}

Although the best method to localize a quench origin is use of voltage taps [1], the method is not desirable for production magnets because of the risks associated with mounting voltage taps. A technique which uses a set of pick-up coils has been developed in order to localize a quench origin without using voltage taps. The technique was originally developed at CERN, and used to localize quench origins of LHC dipole magnets [2,3]. A similar method was then developed at SSCL in order to measure quench locations for SSC dipole magnets. The method developed at SSCL relies on a set of pick-up coils which are sensitive to higher order multipole fields, e.g. quadrupole or sextupole fields, but not sensitive to the main dipole field[4]. Quench antennas relying on the same method were developed for RHIC dipole magnets, and are now being used routinely during the quench tests of the RHIC production dipole magnets. Quench antennas for quadrupole magnets were also developed. The antennas contain pickup coils which are sensitive to sextupole and octupole fields, but not sensitive to the main quadrupole fields. Recently, one of the antennas, being used for $13 \mathrm{~cm}$ aperture interaction region (IR) quadrupole magnets [5], was tested with a magnet which is heavily instrumented with voltage taps. It was confirmed that the quench locations derived from the voltage taps and the quench antenna agreed with each other. This paper briefly describes the antenna system and then presents quench antenna and voltage tap data taken on this magnet.

\section{SYSTEM CONFIGURATION}

The antenna used for the IR magnets consists of two coil arrays which are lined up along the magnet length. Each coil array contains four pickup coils; the first one is sensitive to normal sextupole, the second to skew sextupole, the third to normal octupole, and the fourth to skew octupole field. Figure la presents a cross-sectional view of a coil array. A three dimensional view of a single turn sextupole coil is shown in Figure 1b. It was shown by Morgan [6] that this kind of winding is primarily sensitive to the sextupole field. The octupole coil is similar to the sextupole coil except the wire crosses the coil form every $\pi / 4$. The skew coils are rotated from normal coils by $\pi / 6$ for sextupole and $\pi / 8$ for octupole.

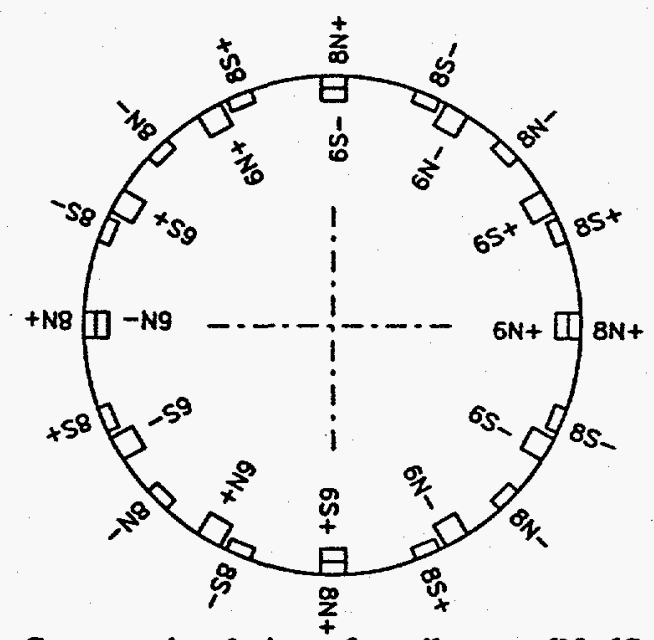

Figure la Cross-sectional view of a coil array. $6 \mathrm{~N}, 6 \mathrm{~S}, 8 \mathrm{~N}$, and $8 S$ indicate normal sextupole, skew sextupole, normal octupole, and skew octupole coil, respectively.

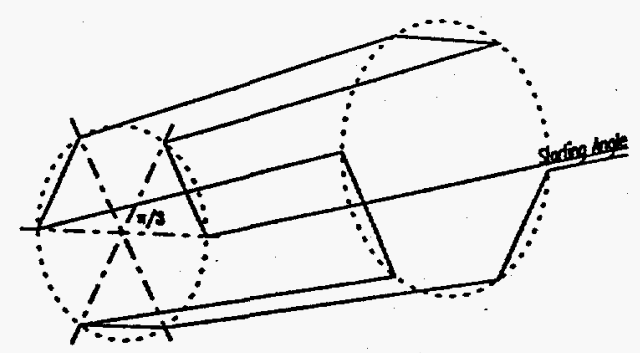

Figure Ib Three dimensional view of a single turn sextupole coil. 
The length of each coil is $150 \mathrm{~mm}$, and the diameter is $79 \mathrm{~mm}$. The centers of the two coil arrays are located 155 $\mathrm{mm}$ from the center of the magnet toward the ends. The coil array close to the lead end is named C-1 and the other is $\mathbf{C}-2$. The number of turns in each windings in the coil array $\mathrm{C}-1$ is 8 , and the number in $\mathrm{C}-2$ is 4 .

\section{MEASUREMENT RESULTS}

The example shown here is the first spontaneous quench taken on magnet QRI998. The quench current is $6745 \mathrm{~A}$, at which the expected gradient is $63.2 \mathrm{~T} / \mathrm{m}$. The quench started in the pole turn, where the voltage taps are instrumented such that terminal voltages of the straight section, the lead end, and the return end can be monitored. The length of the straight section of the magnet is $1309 \mathrm{~mm}$. The voltage taps are mounted slightly inwards so that the length between the taps is $966 \mathrm{~mm}$.

\section{A. Axial Quench Localization}

Figure 2a presents signals of the normal sextupole coils in both arrays in comparison to voltage tap signals of the straight section and both ends of the quenched turn. The signals of the straight section voltage taps and coil C-1 appear to take off almost simultaneously indicating that the quench started in the region where C-1 is located. Following these, the signals of the lead end voltage taps and the coil C-2 take off almost simultaneously. This indicates that the quench origin is halfway between the lead end tap and coil C-2.

More detailed quench propagation analysis can be performed using the voltage tap signal of the straight section. Figure $2 b$ shows quench front locations as a function of time. The locations of antenna coil arrays and the end taps are also indicated at the time when each signal takes off. The plot is derived from the voltage tap signal of the straight section. The voltage increase due to the temperature rise is compensated using the following equation:

$$
V_{c}(t)=V_{s s}(t)-\int_{t_{q 1}}^{t} \frac{V_{s s}\left(t_{q} 3+t-\tau\right)-V_{s s}\left(t_{q}\right)}{V_{s s}\left(t_{q}\right)} \frac{d V_{s s}(\tau)}{d \tau} d \tau
$$

where $V_{c}$ is the compensated voltage, $V_{s s}$ is the raw voltage of the straight section, $t_{q 1}$ is the time when the voltage of the straight section takes off, and $t_{q 3}$ is the time when the return end voltage starts to rise. The size of the normal zone in the straight section is assumed to increase in proportion to $V_{c}$ toward both ends until the quench front reaches the lead end, and after that only toward the return end. The quench start location is derived so that the plot is
Consistent with the times when the voltage tap signals of both ends take off. The plot starts at the lead end edge of $C$ 1 , and then passes through the lead end edge of C-2. This indicates that the antenna signals are in good agreement with the axial quench propagation derived from the voltage tap signals.

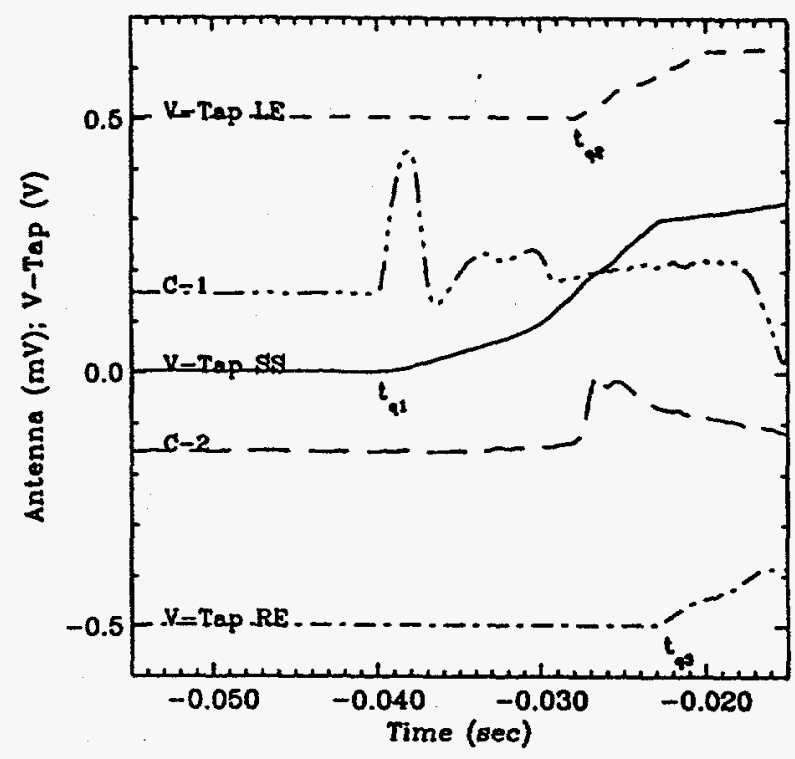

Figure 2a Measured signal of antenna coils and voltage taps. Some of the signals are offset.

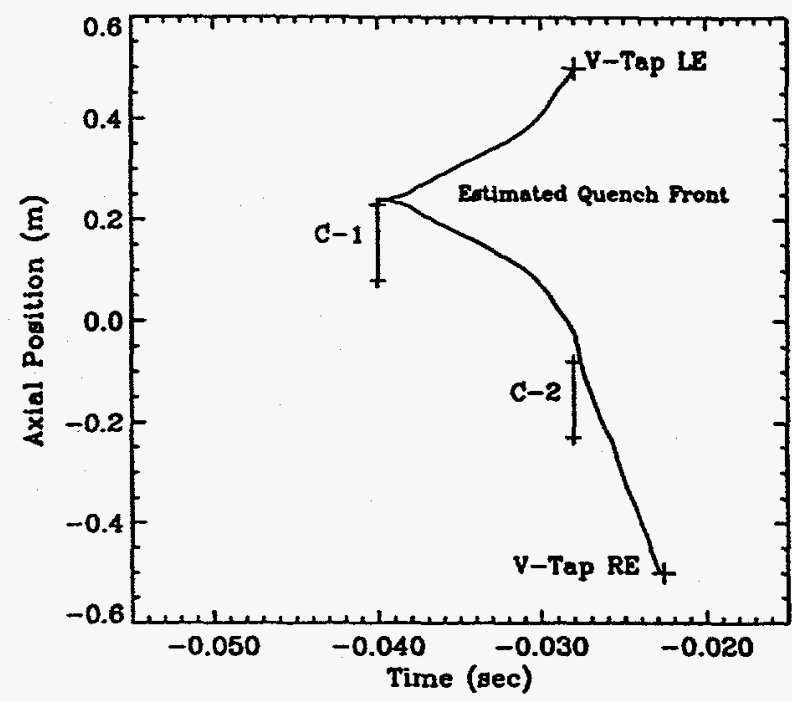

Figure $2 b$ Axial quench localization.

\section{B. Azimuthal Quench Localization}

Figure 3a presents signals of the four coils in the coil array C-1. The signal is believed to be caused by a field distortion due to current redistribution in the quenching turns. Following the analysis performed in Reference 4, we assume that the field distortion is produced by a moving current line. Let us define a Cartesian coordinate system 


\section{DISCLAIMER}

This report was prepared as an account of work sponsored by an agency of the United States Government. Neither the United States Government nor any agency thereof, nor any of their employees, make any warranty, express or implied, or assumes any legal liability or responsibility for the accuracy, completeness, or usefulness of any information, apparatus, product, or process disclosed, or represents that its use would not infringe privately owned rights. Reference herein to any specific commercial product, process, or service by trade name, trademark, manufacturer, or otherwise does not necessarily constitute or imply its endorsement, recommendation, or favoring by the United States Government or any agency thereof. The views and opinions of authors expressed herein do not necessarily state or reflect those of the United States Government or any agency thereof. 


\section{DISCLAIMER}

Portions of this document may be illegible in electronic image products. Images are produced from the best available original document. 
such that the z-axis is parallel to the coil array and the origin is at the center of the coil array. The current $I_{s}$ parallel to the $z$-axis positioned at $(x, y)=r_{s} e^{i \alpha}$, moving with velocity $\left(v_{x}, v_{y}\right)=v_{s} e^{i \beta}$, induces voltages approximated as,

$$
\begin{aligned}
& V_{6 n}=N_{6 n} \frac{3 r_{6 n}{ }^{3} \mu_{0} L_{6 n} I_{s} v_{s}}{\pi r_{s}{ }^{4}} \cos (-4 \alpha+\beta) \\
& V_{6 s}=N_{6 s} \frac{3 r_{6 s}{ }^{3} \mu_{0} L_{6 s} I_{s} v_{s}}{\pi r_{s}{ }^{4}} \sin (-4 \alpha+\beta) \\
& V_{8 n}=N_{8 n} \frac{4 r_{8 n}{ }^{4} \mu_{0} L_{8 n} I_{s} v_{s}}{\pi r_{s}{ }^{5}} \cos (-5 \alpha+\beta) \\
& V_{8 s}=N_{8 s} \frac{4 r_{8 s}{ }^{4} \mu_{0} L_{8 s} I_{s} v_{s}}{\pi r_{s}^{5}} \sin (-5 \alpha+\beta)
\end{aligned}
$$

where the subscripts $6 n, 6 s, 8 n$, and $8 s$ used for the voltage $V$, the number of turns $N$, the length $L$ and the radius $R$, refer to the normal sextupole, skew sextupole, normal octupole, and skew octupole coil, respectively. The system of equations allows us to obtain four unknowns, $r_{s}, \alpha, I_{s} v_{s}$, and $\beta$, analytically. Figure $3 \mathrm{~b}$ summarizes these four values derived for the range indicated by a pair of dotted

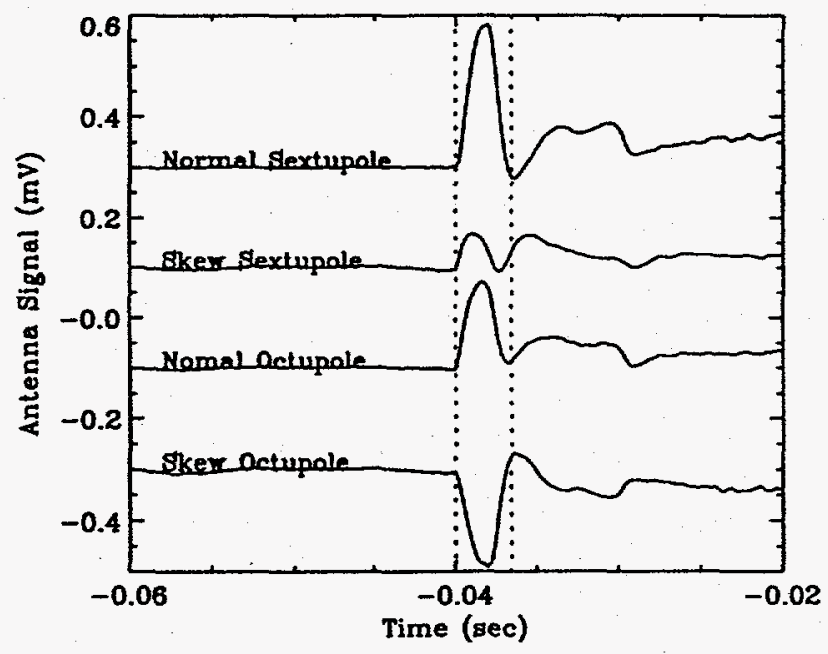

Figure 3a Signals of the coils in C-1. The signals are offset.

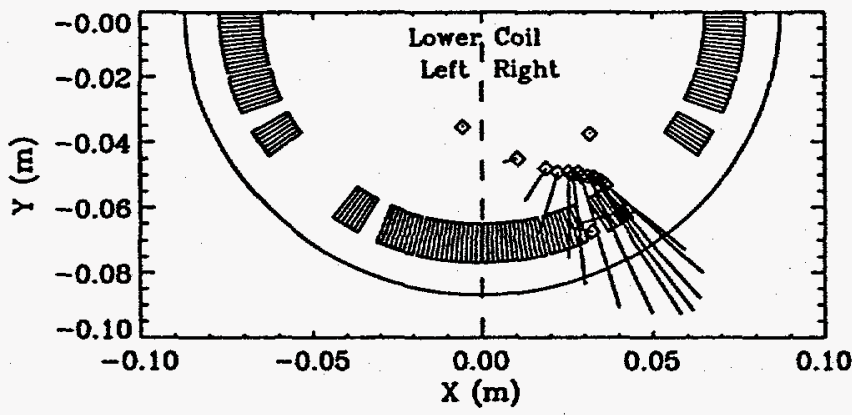

Figure $3 b$ Azimuthal quench localization, as viewed from the lead end. lines in Figure 3a. The diamonds present the position given by $r_{s}$ and $\alpha$ and length and direction of the lines indicate $I_{s} v_{s}$ and $\beta$, respectively. The diamonds are mostly located close to the left pole turn of the lower right coil. The voltage tap signals show that the quench starts in the straight section of the left pole turn in the lower right coil. Although the analysis used here is based on a crude two dimensional model, the azimuthal quench localization result appears to be consistent with the voltage tap result.

This kind of analysis has been performed for most of the quenches of this magnet. In many cases the results are more scattered than the case shown here due to bad signalto-noise ratios. Averaging the data over a range covering the first peak and using the least squares technique, but relying on a similar two dimesional model, a quench origin was determined within several turns of cable of the actual location in most of the cases. Although the two dimensional model is sufficient for the azimuthal quench localization, three dimensional analyses should be performed in order to determine the causes of the signals.

\section{CONCLUSION}

The quench antenna tests made on the RHIC IR quadrupole magnets showed that quench localization based on the quench antenna is consistent with that based on the voltage taps. This proves that the quench antenna introduced here is an adequate device for quench localization in quadrupole magnets.

\section{REFERENCES}

[1] A. Devred, et al., "Quench Start Localization in Full-Lenght SSC R\&D Dipoles," Supercollider-1, M. McAshan ed., Plenum Press, N.Y., 1989, p.73.

[2] D. Leroy, et al., "Quench Observation in LHC Superconducting One Meter Long Dipole Model by Field Perturbation Mesurements," IEEE Trans. on A.S.C., Vol.3 No.1, 1993, p.781.

[3] A. Siemko, et al., "Quench Localization in the Superconducting Model Magnets for the LHC by Means of Pick-up Coils," presented at A.S.C., Boston, 1994.

[4] T. Ogitsu, et al., "Quench Antenna for Superconducting Particle Accelerator Magnets," IEEE Trans. on A.S.C., Vol.3 No.1, 1993, p.781.

[5] G. Gupta, et al., 'Large Aperture Quadrupoles for RHIC Interaction Regions" Proc. 1993 PAC, p. 2745.

[6] G. Morgan "Stationary Coil for Measuring the Harmonics in Pulsed Transport Magnets," Proc. of 4th Int. Conf. on Mag. Tech., BNL, Upton NY, USA, Sep. 19-22, 1972 , p. 787. 